\title{
Valorization of the aqueous phase obtained from hydrothermally treated Dunaliella salina remnant biomass
}

\author{
Kristin Pirwitz ${ }^{\mathrm{a}}$, Liisa Rihko-Struckmannn ${ }^{\mathrm{a}, *}$, Kai Sundmacher ${ }^{\mathrm{a}, \mathrm{b}}$ \\ ${ }^{a}$ Max Planck Institute for Dynamics of Complex Technical Systems, Process Systems \\ Engineering, Sandtorstr. 1, 39106 Magdeburg, Germany. \\ ${ }^{b}$ Otto-von-Guericke-University Magdeburg, Process Systems Engineering, \\ Universitätsplatz 2, 39106 Magdeburg, Germany.
}

\begin{abstract}
Up to $90 \%$ of Dunaliella salina biomass remains unused after extraction of the main product $\beta$-carotene. The potential of mild hydrothermal liquefaction (HTL) to exploit this biomass as a source of valuable byproducts was assessed. The results indicate that $80 \%$ of the remnant was converted into glucose by mild $\mathrm{HTL}\left(100^{\circ} \mathrm{C}, 0 \mathrm{~min}\right)$. The recovered glucose was successfully used as a carbon source to cultivate biotechnologically relevant microorganisms, namely Chlorella vulgaris, Escherichia coli and Saccharomyces cerevisiae. Furthermore, the analysis of energy demand and operating costs confirms the beneficial effect of mild liquefaction on the overall process economics of algal $\beta$-carotene production.
\end{abstract}

Keywords: Dunaliella, Hydrothermal liquefaction, By-product, Carbon source, Process analysis

\footnotetext{
${ }^{*}$ Corresponding author. E-mail address: rihko@mpi-magdeburg.mpg.de, Tel. +49 391 6110 318, Fax: +493916110353.
} 


\section{Introduction}

2 In recent years, hydrothermal liquefaction (HTL) has become an attractive 3 subject of research, especially in the area of microalgal biofuel generation 4 (Orfield et al., 2014). The approach has the clear advantage to use water as 5 reactant, allowing high moisture contents of the biomass (Yang et al., 2004).

6 In microalgal processes biomass dewatering is one of the most cost-intensive 7 steps in the production. Thus, product extraction technologies which oper9 yield under typical HTL conditions is significantly higher compared to that of

conventional extraction because carbohydrates and proteins are partly converted into organic solubles as well (Frank et al., 2013; Delrue et al., 2013). In general, liquefaction leads to the hydrothermal conversion of solid biomass into a bio-crude, an aqueous and a gas fraction. Furthermore, also unconverted biomass remains as solid residue. The reaction is operated at high temperatures of $300-350^{\circ} \mathrm{C}$ and pressures of 5-20 MPa (Chen et al., 2015). However, various studies demonstrated significant product yields even under milder conditions (e.g. Gai et al. (2015); Minowa et al. (1995)). During the time course of liquefaction, the initial hydrolysis and depolymerization compete with repolymerization at a later stage (Gai et al., 2015). The fractions obtained by the hydrothermal treatment are strongly dependent on the biochemical composition of the applied biomass (Biller and Ross, 2011). Nevertheless, the study of Yu et al. (2011) revealed, that even low-lipid biomass can be attractive for bio-oil production, which is the main product of interest of the liquefaction. However, there are various other valuable components 
found in the product fractions, e.g. nutrients, organic acids, alkanes, alkenes, cyclic ketones and phenols and nitrogenous organic compounds (Brown et al., 2010; Biller and Ross, 2011; Pham et al., 2013). To achieve an optimal valorization of the product fractions of HTL, researchers are looking for possible applications of the aqueous phase obtained in liquefaction besides the already exploitable bio-oil phase. One successfully investigated approach is the nutrient recycling from the aqueous phase for microalgae cultivation (López Barreiro et al., 2015; Hognon et al., 2015; Biller et al., 2012).

The green microalga D. salina is an industrially used production organism of natural $\beta$-carotene. After extraction of the pigment up to $90 \%$ biomass remains unexploited in the process. The valorization of this remnant biomass can improve the overall process economics significantly. With $\beta$-carotene extraction by organic solvents, the main fraction of triglycerides in the biomass is extracted as well. Thus, conventional liquefaction of the remnant biomass seems not to be promising for lipid-based biofuel production at the first glance. As the alga has no rigid cell wall, cell constituents are easily accessible and due to the small cell size a rapid heat transfer during liquefaction is possible. This could facilitate the release of other valuable products from the remnant biomass. The present work aims for the assessment of mild HTL of extracted D. salina biomass. Initially, the macromolecular and elemental biomass composition was determined to identify possible liquefaction products. Under consideration of the process economics, moderate temperatures between $100-200^{\circ} \mathrm{C}$ were investigated in the absence of a catalyst. Thereafter, the recovered glucose-containing aqueous phase was used as carbon source for mixotrophic or heterotrophic cultivations of three different mi- 
croorganisms. One of the main challenges of HTL is the considerable energy consumption due to the high operation temperatures and pressures used in the process. Therefore, energy consumption and operating costs for the applied liquefaction condition of D. salina were calculated to finally evaluate the results.

\section{Materials and Methods}

\subsection{Origin and composition of the biomass}

D. salina biomass was purchased as a carotenoid-containing dried powder from Denk Ingredients GmbH, Germany (Art. no: 967996). Prior to hydrothermal treatment, pigments were extracted to get remnant biomass. The extraction was carried out for $5 \mathrm{~h}$ using a Soxhlet extractor and n-hexane as extraction solvent. After the solvent has been evaporated in a rotary evaporator, the concentrated extract as well as the extracted biomass were dried overnight, respectively. The lipid content of the raw biomass was estimated from the weight of the dried, solvent free extract. The fraction of carotenoids in the biomass was measured spectroscopically using the protocol of Lichtenthaler (2001).

The carbon, hydrogen, nitrogen and sulfur (CHNS) contents in the remnant biomass were analyzed by elemental analysis (Currenta, Germany). Moisture and ash contents of the extracted D. salina powder were determined by weight difference of samples prior and after overnight drying at $100{ }^{\circ} \mathrm{C}$ and $450^{\circ} \mathrm{C}$, respectively. The concentration of carbohydrates was quantified based on glucose by an enzymatic test kit (R-Biopharm AG, Germany). The method of Lowry was used for protein content determination (Lowry et al., 
1951).

\subsection{Mild hydrothermal liquefaction of remnant biomass}

A $200 \mathrm{~mL}$ stainless steel batch reactor (Picoclave 3, Büchi Labortechnik GmbH, Germany) was used to hydrothermally liquefy the D. salina biomass. Therefore, a slurry containing $6 \mathrm{~g}$ of the extracted biomass mixed with $100 \mathrm{~mL}$ bidistilled water was placed in the reactor. After sealing the reactor, the headspace was purged by nitrogen for 5 min to remove air. During an experiment the suspension was mixed with a frequency of $1800 \mathrm{rpm}$. The reactor was operated at temperatures and reaction times between $100-200{ }^{\circ} \mathrm{C}$ and 0-60 min, respectively. Once the set point of temperature was reached, the reaction time was registered. For 0 min reaction time, the heating process was directly stopped after reaching the set temperature. The time courses of temperature, pressure and stirrer frequency were recorded (Büchi log'n see bls2, Büchi Labortechnik GmbH, Germany). After cooling down, the reactor content was transferred through a preweighted filter into a separation funnel. To collect any remaining lipophilic products, the reactor and stirrer were rinsed with $60 \mathrm{~mL}$-hexane. Afterwards, the n-hexane mixture was passed through another preweighted filter into the separation funnel containing the aqueous phase. Filters were dried and oil residuals in the filter and on the solid surface were recovered by applying 30 min Soxhlet extraction using $60 \mathrm{~mL}$ n-hexane. Afterwards, filters were dried again and weighted to determine the yield of the solid phase. The immiscible water-hexane system in the separation funnel was intensively mixed to extract all bio-oil products into the hydrophobic phase. After that, the biphasic mixture was allowed to separate into an aqueous and a hydrophobic n-hexane phase. To recover the 
bio-crude the n-hexane phase was mixed with that obtained during Soxhlet extraction of the filters and evaporated at $40{ }^{\circ} \mathrm{C}$ and reduced pressure. For the quantification of the bio-crude fraction the remaining lipophilic substances were dried overnight. The yields of all product fractions were calculated based on the dry weight of the used biomass. The yield of the aqueous phase was determined by weighting two $6 \mathrm{~mL}$ samples of the aqueous phase after overnight drying. The yield of the gas phase was calculated as subtraction of the yields of aqueous, solid and bio-crude phase from $100 \%$. Since the yield of the aqueous phase clearly exceeded that of the other phases, detailed investigation was done to identify its chemical compounds.

\subsection{Analysis of the aqueous phase}

Concentrations of glucose, fructose, sucrose, galactose and glycerol were determined in duplicates or triplicates using substrate specific enzymatic test kits (R-Biopharm AG, Germany) based on absorbance measurements at $340 \mathrm{~nm}$. Nutrient concentrations were determined by ion chromatography (930 compact IC flex, Metrom, Switzerland). Therefore, concentrations of anions were measured using a Metrosep A Supp 5 column at $35^{\circ} \mathrm{C}$, an eluent containing $3.2 \mathrm{mM} \mathrm{Na}_{2} \mathrm{CO}_{3}$ and $1 \mathrm{mM} \mathrm{NaHCO}_{3}$ and a flow rate of $0.7 \mathrm{~mL} \mathrm{~min}^{-1}$. Cations were measured using a Metrosep C6 column at $45^{\circ} \mathrm{C}$, an eluent containing $1.7 \mathrm{mM} \mathrm{HNO}_{3}$ and $1.7 \mathrm{mM} \mathrm{C}_{7} \mathrm{H}_{5} \mathrm{NO}_{4}$ and a flow rate of $0.9 \mathrm{~mL} \mathrm{~min}^{-1}$. 
2.4. Cultivation of different microorganisms on glucose obtained from the aqueous phase

Cultivation experiments were conducted using microbial representatives of highly relevant production organisms in biotechnology, which are already industrially applied for the production of a wide range of products (see Section 3.5 for more details). Chlorella vulgaris SAG 211.12, Escherichia coli MG1655 and Saccharomyces cerevisiae Y187 were used as model organisms for algae, bacteria and yeast, respectively. C. vulgaris was grown mixotrophically at a pH of 7.1 in $300 \mathrm{~mL}$ shaking flasks containing $100 \mathrm{~mL}$ BG11 medium (Stanier et al., 1971) with $0.5 \%$ glucose. The cultivations were carried out in a rotary shaking incubator as previously described in Pirwitz et al. (2015b). E. coli was cultivated aerobically in $500 \mathrm{~mL}$ shaking flasks filled with $75 \mathrm{~mL}$ LB medium (tryptone $1 \%$, yeast extract $0.5 \%$, sodium chloride $0.5 \%$, glucose $0.5 \%$ ) adjusted to a $\mathrm{pH}$ of 7 . The cultivation occurred at $37^{\circ} \mathrm{C}$ and a mixing frequency of $200 \mathrm{rpm}$. Growth experiments with S. cerevisiae were carried out under aerobic condition using $500 \mathrm{~mL}$ shaking flasks filled with $100 \mathrm{~mL}$ YPD medium (tryptone $2 \%$, yeast extract $1 \%$, glucose $2 \%$ ). The cultures were incubated at $30^{\circ} \mathrm{C}$ and $200 \mathrm{rpm}$.

In preparation of all cultivations, the respective glucose concentration (see above) in the control media was adjusted by addition of purchased glucose (Sigma-Aldrich, USA). The glucose concentration in the individual test medium was adjusted by adding appropriate volumes of the aqueous phase ( $\sim 48 \mathrm{~g} \mathrm{~L}^{-1}$ ) obtained by mild $\mathrm{HTL}\left(100^{\circ} \mathrm{C}, 0 \mathrm{~min}\right)$ to reach concentration equal to the corresponding control medium. That means that approximately $10 \mathrm{~mL}$ aqueous phase per $100 \mathrm{~mL}$ LB as well as BG11 or $42 \mathrm{~mL}$ 
per $100 \mathrm{~mL}$ YPD were added to the medium before the water and $\mathrm{pH}$ adjustment was done. All other media ingredients were identical in source and concentration to the above described control media recipes. The $\mathrm{pH}$ in the control and test media was adjusted to the same value.

The growth of all microorganisms was followed by absorbance measurements of the cultures at $735 \mathrm{~nm}$ for $C$. vulgaris or $600 \mathrm{~nm}$ for E. coli and S. cerevisiae. The glucose consumption was determined in filtrated supernatants of the cultures by the previously mentioned enzymatic assay kit (see Section 2.3).

\subsection{Energy and operating cost analysis of hydrothermal biomass conversion}

\subsubsection{Process model description}

To calculate the additional energy demand and operating costs for the glucose generation from remnant biomass, the process model described by Pirwitz et al. (2015a) was extended by the process unit of liquefaction. The energy consumption of liquefaction comprised the energy required for water and slurry pumping, for mixing as well as for heating. Pumping and mixing work was calculated according to the assumptions made in the process model (Pirwitz et al., 2015a). The energy required for the heating of the algal slurry containing $6 \%$ dry weight biomass was estimated by the heat capacity equation:

$$
Q=c_{p_{i}} \cdot \Delta T \cdot m_{i}
$$

where $Q$ is the heat energy required in $\mathrm{kJ}, c_{p_{i}}$ is the heat capacity of the component $i$ (water or algae biomass) in $\mathrm{kJ} \mathrm{kg}^{-1} \mathrm{~K}^{-1}, \Delta T$ is the temperature 
change in $\mathrm{K}$ and $m_{i}$ is the mass of species $i$ in $\mathrm{kg}$. Liquefaction was assumed to be operated in a continuous, isolated reactor with a working volume of $400 \mathrm{~L}$. The reactor was simulated to be heated from $20^{\circ} \mathrm{C}$ to $100^{\circ} \mathrm{C}$ by a conventional boiler in combination with a heat exchanger with an efficiency of $80 \%$ (Delrue et al., 2013). The heat capacity of algal biomass was set to the value $1.25 \mathrm{~kJ} \mathrm{~kg}^{-1} \mathrm{~K}^{-1}$ (Orosz and Forney, 2008). After liquefaction the reaction mixture was separated in a separation unit. The biomass concentration as well as the biomass conversion and the yield of glucose were adopted from the results of the mild HTL experiment at $100^{\circ} \mathrm{C}$ presented in this study. The total revenue of glucose was estimated considering the recent commodity price of $747.55 \mathrm{USD} \mathrm{t}^{-1}$ published by the United States Department of Agriculture (Department of Agriculture, 2016).

\subsubsection{Statistics}

For the consideration of uncertainties of the parameter values used in the process model, Monte Carlo simulation was applied using $5 \times 10^{5}$ independent normally distributed samples to analyze the impact on the predictions. The variances were defined in dependence of the used parameters. A variance of $\sigma_{\text {parameter }}^{2}=\left(0.25 / 3 \mu_{\text {parameter }}\right)^{2}$ was assumed for parameters derived from literature, which is 3 standard deviations corresponding to $25 \%$ of the nominal parameter. For the determined experimental parameters, the observed experimental variances were used. The simulations of the process proposed model were done using Matlab (MathWorks). 


\section{Results and discussion}

\subsection{Biomass composition}

One crucial factor affecting the product yields of HTL is the composition of the biomass. Therefore, the biochemical and elemental compositions of the used D. salina powder were analyzed (see Table 1 ). In the following, the optional uses of the remnant biomass are considered shortly.

Lipids and carotenoids in the biomass were removed by initial extraction, making the remnant unattractive as a source of lipid-based fuels or unsaturated fatty acid at first sight. However, the works of Biller and Ross (2011) and Yu et al. (2011) demonstrate, that the liquefaction of low-lipid containing biomass can also lead to efficient bio-oil production since the source of bio-oil in the biomass is not only the lipid fraction but also the protein and carbohydrate fractions. Thus, it seems possible to achieve adequate bio-oil yields even with the extracted biomass.

Due to the need of nitrogen deprivation during $\beta$-carotene production in $D$. salina, the biomass is low in nitrogen $(<0.2)$ and consequently has a low protein content $(<9 \%)$. It is therefore not highly feasible to use the remnant biomass as protein source for animal feed. Another approach, also reported in literature, is the recycling of nutrients recovered from the aqueous phase after liquefaction into the cultivation unit (López Barreiro et al., 2015; Hognon et al., 2015). However, in the present work the ash content, and thus the mineral salt content is negligible, reducing the potential for sustainable nutrient recycling.

The heating value of the extracted biomass was calculated according to the Boie equation (Boie, 1953). With a value of $17.32 \mathrm{MJ} \mathrm{kg}^{-1}$ it is low com- 
pared to lignocellulosic biomass or coal, but similar to other algal feedstocks (Daneshvar et al., 2012; Chen et al., 2015). Accordingly, direct combustion of the biomass comparable to lignocellulose containing feedstocks does not seem to be promising. Interestingly, with $85.6 \%$ the carbohydrate content of the used D. salina remnant is remarkably high which once again can be explained by the nitrogen deprivation under production conditions. In this state, proteins in the biomass are decomposed and lipids as well as carbohydrates serve as storage molecules for cell maintenance. As the major fraction of the storage lipids was removed by extraction, the remnant biomass mainly consists of carbohydrates. This macromolecule class can serve as precursor of fine chemicals and fuels.

All in all, two potential approaches to valorize the residual biomass need to be further investigated. On the one hand, there is the possibility to achieve satisfactory biofuel yields comparable to the above mentioned low-lipid biomass. On the other hand, in terms of process economics the extraordinary high carbohydrate content of the biomass seems to be the most promising by-product in the overall process.

\subsection{Influence of the reaction time on $H T L$ yields}

To examine the impact of the reaction time on the yields of the gas, solid, bio-crude and aqueous fractions, liquefaction experiments were carried out for 0,30 and $60 \mathrm{~min}$ at 160,180 and $200^{\circ} \mathrm{C}$, respectively (see Fig. 1). There is no clear correlation between the reaction time and the product yield. For the liquefaction at $180^{\circ} \mathrm{C}$ and $200^{\circ} \mathrm{C}$, a slight increase of the bio-oil phase was visible while at the same time the aqueous phase yield declined marginally. 
A similar behavior was described by Yu et al. (2011) for the low-lipid alga Chlorella pyrenoidosa. With respect to the biomass conversion, the liquefaction experiment at $200^{\circ} \mathrm{C}$ resulted in a decrease with prolonged reaction time, showing possible initiation of repolymerization. During longer reaction times, the repolymerization reaction starts to compete with the initial hydrolysis and depolymerization processes and leads to higher solid yields (Gai et al., 2015). In all cases, most of the biomass was converted into aqueous phase components regardless of the reaction time. With increasing temperature and time, the color of the aqueous phase turned from light yellow into deep brown (data not shown). In parallel, the solid yield slightly increased to a maximum of up to $8 \%$. These observations could be a first indication of a high sugar content in this phase as sugars tend to visibly oxidate at higher temperatures. For comparison, in the study of Biller and Ross (2011) a solid yield of up to $20 \%$ was attained after HTL of the model compounds glucose and starch, demonstrating that carbohydrates are partly converted into solids during HTL.

\subsection{Influence of the reaction temperature on HTL yields}

The impact of the reaction temperature on the yields of the product fractions is illustrated in Fig. 2. The temperature was varied from 100 to $200{ }^{\circ} \mathrm{C}$ in $20^{\circ} \mathrm{C}$ intervals. The reaction time was constantly fixed at $0 \mathrm{~min}$, which means that the heating process was directly stopped after reaching the set reaction temperature. Obviously, the yield of the solid phase declined from $15 \%$ to $5 \%$ with increasing temperature. In contrast, the gas fraction increased in line with the temperature which can be explained by more intensive hydrothermal gasification at higher temperatures (Gai et al., 2015). 
Both observations are characteristic for hydrothermal liquefaction of microalgal biomass (López Barreiro et al., 2014; Yu et al., 2011). Nevertheless, in all experiments, the yield of the gas fraction was relatively low. $2-6 \%$ of the extracted biomass was converted into biocrude by mild HTL which is not sufficient for the purpose of biofuels. This phenomenon can be attributed to the previously mentioned degradation of macromolecules like proteins or carbohydrates during the hydrothermal treatment (Biller and Ross, 2011). For example, Yang et al. (2015) demonstrated a conversion of up to $5 \%$ of pure polysaccharides into bio-oil during liquefaction which is comparable to the results achieved in our study by liquefying the carbohydrate-rich biomass. The yield of the aqueous phase was constantly in the range of 80$90 \%$. Thus, it represents the main product of the hydrothermal treatment of the residual D. salina biomass.

The reaction temperatures investigated in the present study are below the commonly used HTL temperatures ranges of 200 to $400{ }^{\circ} \mathrm{C}$ (Hognon et al., 2015; Chen et al., 2015; Toor et al., 2013). In the case of D. salina, cell disruption as well as biomass decomposition requires less energy input than that of other microalgae species, due to the lack of a rigid cell wall. Even at a reaction temperature of $100^{\circ} \mathrm{C}$ a biomass conversion level of $87 \%$ was achieved (see Fig. 2). At increased temperature the conversion could be further improved by $10 \%$. These results are contrary to the low HTL conversion of $49 \%$ reached by Yang et al. (2011) for a lipid-rich D. salina at $200^{\circ} \mathrm{C}$. Accordingly, the pretreatment by lipid extraction used in the present work seems to improve the efficiency of mild liquefaction for $D$. salina biomass. 


\subsection{Products of the aqueous phase}

Based on the results from Sections 3.2 and 3.3, an efficient recovery of valuable compounds from the aqueous phase of remnant biomass seems to be promising. Even at low temperatures, high amounts of solubles were released into the aqueous phase. However, the products of interest and their value need to be identified and quantified to assess the economic feasibility of the approach.

The nutrient content of the aqueous phase was analyzed by ion chromatography (see Table 2 and 3). Anion as well as cation concentrations were detectable only in traces and low compared to the results obtained with other microalgal species (Biller et al., 2012; López Barreiro et al., 2015). The low nitrogen content of the biomass was reflected in the negligible concentrations of $\mathrm{NH}_{4}^{+}, \mathrm{NO}_{2}^{-}$and $\mathrm{NO}_{3}^{-}$. Thus, the unfeasible use of the aqueous phase as protein source was once again confirmed. The crucial nutrients of the culture medium, namely, $\mathrm{Na}^{+}, \mathrm{K}^{+}, \mathrm{Ca}^{2+}, \mathrm{Mg}^{2+}, \mathrm{SO}_{4}{ }^{2-}, \mathrm{Cl}^{-}$and $\mathrm{PO}_{4}{ }^{3-}$, were detected in concentrations not higher than $0.01 \mathrm{wt} \%$ of the investigated biomass. Consequently, the recycling of the aqueous phase in the D. salina cultivation unit seems to be unreasonable. Interestingly, in all cases small amounts of organic acids in the form of acetate and formate were identified, showing an increasing tendency in line with temperature and reaction time (see Table 2). Organic acids are metabolites of green algae and can be potentially used as an carbon source for mixotrophic growth (Biller et al., 2012). One expected product in the aqueous phase was the polar molecule glycerol, which is a degradation product of triglycerides and a by-product in the $\beta$-carotene production located in the cytoplasm of D. salina. However, no 
significant concentrations were detected in the samples (data not shown). Regarding the types of carbohydrates, cellulose as well as hemicellulose were unexpected to be present in the D. salina samples, due to the lack of a rigid cell wall. Sucrose, fructose and saccarose were present only in small portions or not detectable in the aqueous phase samples of the present study (see Table S1).

The relatively high carbohydrate content of the biomass (see Table 1) led to the presumption that glucose as primary product of photosynthesis and depolymerization product of the storage molecule starch could be one of the main carbohydrate molecules in the aqueous phase. The measured glucose concentrations confirmed this hypothesis (see Table 4). Even at the lowest temperature a glucose yield of $77 \mathrm{wt} \%$ of the used biomass was reached. The glucose seems to be easily recoverable from the biomass by mild hydrothermal treatment. No clear trend of the glucose yield was visible with the increase of the reaction temperature or time. The high glucose concentrations can be explained by the relatively low reaction temperatures applied in the present work. A further increase of the temperature above $200^{\circ} \mathrm{C}$ would likely lead to a conversion of glucose into other molecules, especially 5-hydroxymethylfurfural (5-HMF) (Srokol et al., 2004). Since glucose itself is a valuable feedstock and carbon source for chemical and biotechnological applications, it was selected as target by-product of the $\beta$-carotene production process. With respect to the process economics, possible applications of the glucose in the aqueous phase generated during liquefaction at $100^{\circ} \mathrm{C}$ were investigated. At this temperature a relatively low energy input is needed to reach a high concentration of glucose. 


\subsection{Glucose from aqueous phase as microbial carbon source}

As glucose is a common organic carbon source for a wide range of microorganisms, diverse applications in microbial production processes can be found. However, the extensive applicability also carries a high risk of undesired contamination for open pond cultivation using glucose as substrate. Consequently, the substrate is more feasible for closed bioreactor cultivations. In the following, the aqueous phase was applied as carbon source to grow three different biotechnologically well-established production organisms.

The bacterium E. coli is one of the most important production organisms in biotechnology. It is currently used for the production of recombinant proteins in pharmaceutical industry and for biomolecular products like amino acids and primary as well as secondary metabolites (Choi et al., 2006). Due to the fact that glucose is one of the main substrates in E. coli fermentation, the ability of the bacterium to consume the liquefaction-derived glucose was investigated (see Fig. 3 a). Therefore, the glucose concentration in the modified cultivation medium was adjusted by addition of the aqueous phase. All other ingredients were added with identical concentrations to the control medium (see Section 2.4). The growth curves demonstrate a similar behavior of the culture cultivated on the control medium compared to that cultivated on the modified medium. The same holds true for the glucose consumption of the bacteria cultures in both media (see Fig. 3 a). Accordingly, there is no inhibitory effect of the aqueous phase components aside glucose which would hamper the growth of E. coli. A similar conclusion was drawn for the use of the aqueous phase from HTL of Nannochloropsis oculata for the cultivation of E. coli as well as Pseudomonas putida (Nelson et al., 2013). 
Both microorganisms revealed an improved final optical density in medium mixed with up to $30 \%$ of the aqueous phase which contained approximately $20 \mathrm{~g} \mathrm{~L}^{-1}$ organic carbon (e.g. in form of glycerol and acetate). In the case of $P$. putida even an increase in growth rate was reached for cultivation in the mixed medium compared to the control medium. In addition, a recently published life cycle analysis of algal liquefaction also demonstrated a clear beneficial effect of the use of the aqueous phase for $E$. coli cultivation on the overall process economics (Orfield et al., 2014).

For the next cultivation experiment we chose $C$. vulgaris as a potential microorganism with high biotechnologically relevance. The green microalga is largely used for mass cultivation to generate biomass especially for nutritional purpose. One important product substance from $C$. vulgaris is $\beta$-1,3-glucan, which serves as immunostimulator (Richmond, 2007). Furthermore, the alga is grown for the production of lipid-rich flour as well as protein-rich powder applied as functional nutrition (Piechocki et al., 2011). Besides photoautotrophic cultivation with $\mathrm{CO}_{2}, C$. vulgaris is cultivated mixotrophically and heterotrophically utilizing an additional organic carbon source like glucose (Richmond, 2007). Fig. 3 b illustrates that C. vulgaris was able to mixotrophically consume the liquefaction-derived glucose in the same manner as the glucose in the control medium. Both cultures grow to a comparable optical density. Similar results were reported by Biller et al. (2012), who cultivated C. vulgaris phototrophically on diluted aqueous phases after liquefaction to recycle nutrients and carbon sources. The alga was able to use the recycled nutrients in a 200-fold diluted aqueous phase comparable to the culture in the standard medium. However, a less diluted aqueous phase resulted 
in an inhibitory effect of algal growth. In the present work, we applied an initial glucose concentration of $5 \mathrm{~g} \mathrm{~L}^{-1}$ which required an approximate 10fold dilution of the aqueous phase. In spite of the high concentration, there was no visible inhibitory effect on mixotrophic growth due to other possible substances derived from the aqueous phase. A possible explanation for the observation of Biller et al. (2012) is the presence of toxic compounds in the aqueous phase of HTL (Gai et al., 2015). The work of Pham et al. (2013) revealed the correlation between cytotoxicity of the aqueous phase from HTL of Spirulina patensis and the presence of nitrogenous organic compounds. These compounds are generally derived from the degradation and repolymerization of carbohydrates and proteins during HTL at temperatures above $200^{\circ} \mathrm{C}$ (Gai et al., 2015). However, the aqueous phase used in the present study was generated by hydrothermal treatment at $100^{\circ} \mathrm{C}$ of low-protein biomass. Accordingly, no inhibitory effects on algal growth by nitrogenous organic compounds are expectable.

In the third experiment, the usability of liquefaction-derived glucose was investigated for the cultivation of $S$. cerevisiae which is currently the most frequently used yeast strain to produce a wide range of commercial platform chemicals (Li and Borodina, 2015). The growth curves in Fig. 3 c indicate a comparable biomass generation and glucose consumption of cultures growing on standard medium and those growing on the modified medium. In contrast to the above mentioned work of Nelson et al. (2013) growth of the yeast strain was not inhibited by the aqueous phase. In this study S. cerevisiae was not likely to be able to consume a variety of different organic carbon sources present in the aqueous phase. This assumption arose from the fact that 
the inhibitory effect decreased by the supplementation of additional glucose. The aqueous phase derived from mild HTL of D. salina in the present work mainly consists of glucose, which seems to be the preferred carbon source of the yeast. With respect to product generation, Pervez et al. (2014) reported an ethanol yield of $84 \%$ using $S$. cerevisiae fermentation with glucose originating from cassava starch by saccharification and liquefaction. Ethanol is one possible product derived from glucose which is largely used as biofuel (Kim et al., 2015).

For all investigated microorganisms, the recovered glucose was successfully applicable which makes it a promising carbon source for biotechnological purpose.

\subsection{Energy and operating cost analysis of glucose production}

To assess the feasibility of liquefaction regarding cost and energy demand the parameters and results of the experimental studies (e.g. biomass concentration, biomass conversion and glucose yields) were integrated into the extended process model for D. salina based $\beta$-carotene production developed by Pirwitz et al. (2015a) (see Section 3.6 for more details). Uncertainties in the assumed and measured parameters were considered via Monte Carlo simulations.

The results of the energy and operating cost analysis for glucose production by mild HTL are depicted in Fig. 4. With a consumption of $102 \mathrm{kWh} \mathrm{d}^{-1}$ the liquefaction needs less energy compared to all other process steps in $\beta$ carotene production (see Fig. 4 a). Only $2.3 \%$ of the overall energy is needed to liquefy the remnant biomass. In detail, the production of one $\mathrm{kg}$ glucose consumes $0.74 \pm 0.14 \mathrm{kWh}$ energy. These results are in line with the 
production cost of glucose (see Fig. 4 b). By avoiding harsh reaction conditions and the use of catalysts, an inexpensive by-product generation seems possible. In detail, the raw production cost (excluding costs of tax and manpower) amount to $0.09 \pm 0.02$ USD per $\mathrm{kg}$ glucose. Using the current market price of glucose (Department of Agriculture, 2016), total revenues of $34036 \pm 3271 \mathrm{USD}^{-1}$ can be achieved for the annual glucose production less the estimated production costs of $4318 \pm 910 \mathrm{USD} \mathrm{a}^{-1}$. However, one should note, that the selling price is calculated on the basis of glucose syrup and is thereby overestimated since the product of the present work is a glucose containing aqueous solution. To achieve syrup consistency further energy is required and thus costs will arise for an additional concentration step. Nevertheless, the results of the cultivation experiments reveal the successfully proven application of the glucose in form of an aqueous solution without the need of further concentration into syrup. Regarding the biomass utilization, it became obvious that the main part of the low valued remnant was converted into valuable glucose (see Fig. 4 c). More specifically, the generation of $45.5 \mathrm{t}$ glucose per year can be achieved by liquefying the annual produced biomass remnant of $59.1 \mathrm{t}$ according to the results of the modeled $\beta$-carotene production process. Thus, the by-product valorization by mild HTL is highly beneficial for the overall process economics and a holistic biorefinery concept with a more extensive exploitation of available biomass components appears possible. 


\section{Conclusion}

In the present work the potential of HTL to use remnant D. salina biomass was investigated. Mild process conditions of $100^{\circ} \mathrm{C}$ and 0 min reaction temperature and time were found to be sufficient to reach high biomass conversion levels of at least $85 \%$. In the aqueous phase, glucose was the most abundant product which was successfully used to grow three different microorganisms of biotechnologically relevance. Consequently, liquefaction-derived glucose can be used as valuable by-product in the $\beta$-carotene production process. The beneficial effect of the overall process economics was clearly verified by the calculation of energy demand and operating costs.

\section{Acknowledgments}

This research work was partly supported by the Center for Dynamic Systems (CDS) funded by the Federal State Saxony-Anhalt (Germany). The authors would like to thank N. Hellmold, M. Ikert and A. Reichelt for technical assistance in performing the experiments. 


\section{References}

Department of Agriculture, U.S., 2016. Sugar and sweeteners yearbook tables. Access: 25.01.2016, www.ers.usda.gov/data-products/sugar-andsweeteners-yearbook-tables.aspx.

Biller, P., Ross, A.B., 2011. Potential yields and properties of oil from the hydrothermal liquefaction of microalgae with different biochemical content. Bioresour. Technol. 102, 215-225.

Biller, P., Ross, A.B., Skill, S.C., Lea-Langton, A., Balasundaram, B., Hall, C., Riley, R., Llewellyn, C.A., 2012. Nutrient recycling of aqueous phase for microalgae cultivation from the hydrothermal liquefaction process. Algal Res. 1, 70-76.

Boie, W., 1953. Fuel technology calculations. Energietechnik 3, 309-316.

Brown, T.M., Duan, P., Savage, P.E., 2010. Hydrothermal liquefaction and gasification of Nannochloropsis sp. Energ. Fuels 24, 3639-3646.

Chen, W.H., Lin, B.J., Huang, M.Y., Chang, J.S., 2015. Thermochemical conversion of microalgal biomass into biofuels: A review. Bioresour. Technol. 184, 314-327.

Choi, J.H., Keum, K.C., Lee, S.Y., 2006. Production of recombinant proteins by high cell density culture of Escherichia coli. Chem. Eng. Sci. 61, 876885.

Daneshvar, S., Salak, F., Ishii, T., Otsuka, K., 2012. Application of subcriti- 
cal water for conversion of macroalgae to value-added materials. Ind. Eng. Chem. Res. 51, 77-84.

Delrue, F., Li-Beisson, Y., Setier, P.A., Sahut, C., Roubaud, A., Froment, A.K., Peltier, G., 2013. Comparison of various microalgae liquid biofuel production pathways based on energetic, economic and environmental criteria. Bioresour. Technol 136, 205-212.

Frank, E.D., Elgowainy, A., Han, J., Wang, Z., 2013. Life cycle comparison of hydrothermal liquefaction and lipid extraction pathways to renewable diesel from algae. Mitig. Adapt. Strateg. Glob. Change 18, 137-158.

Gai, C., Zhang, Y., Chen, W.T., Zhang, P., Dong, Y., 2015. An investigation of reaction pathways of hydrothermal liquefaction using Chlorella pyreno $i$ dosa and Spirulina platensis. Energ. Convers. Manage. 96, 330-339.

Hognon, C., Delrue, F., Boissonnet, G., 2015. Energetic and economic evaluation of Chlamydomonas reinhardtii hydrothermal liquefaction and pyrolysis through thermochemical models. Energy 93, Part 1, 31-40.

Kim, S.S., Ly, H.V., Kim, J., Lee, E.Y., Woo, H.C., 2015. Pyrolysis of microalgae residual biomass derived from Dunaliella tertiolecta after lipid extraction and carbohydrate saccharification. Chem. Eng. J. 263, 194-199.

Li, M., Borodina, I., 2015. Application of synthetic biology for production of chemicals in yeast Saccharomyces cerevisiae. FEMS Yeast Res. 15, 1-12.

Lichtenthaler, H.K., 2001. Chlorophylls and carotenoids: Measurement and characterization by UV-vis spectroscopy. Current Protocols in Food Analytical Chemistry . 
López Barreiro, D., Bauer, M., Hornung, U., Posten, C., Kruse, A., Prins, W., 2015. Cultivation of microalgae with recovered nutrients after hydrothermal liquefaction. Algal Res. 9, 99-106.

López Barreiro, D., SamorLò, C., Terranella, G., Hornung, U., Kruse, A., Prins, W., 2014. Assessing microalgae biorefinery routes for the production of biofuels via hydrothermal liquefaction. Bioresour. Technol. 174, 256-265.

Lowry, O.H., Rosebrough, N.J., Farr, A.L., Randall, R.J., 1951. Protein measurement with the folin phenol reagent. J. Biol.Chem. 193, 265-275.

Minowa, T., Yokoyama, S.y., Kishimoto, M., Okakura, T., 1995. Oil production from algal cells of dunaliella tertiolecta by direct thermochemical liquefaction. Fuel 74, 1735-1738.

Nelson, M., Zhu, L., Thiel, A., Wu, Y., Guan, M., Minty, J., Wang, H.Y., Lin, X.N., 2013. Microbial utilization of aqueous co-products from hydrothermal liquefaction of microalgae Nannochloropsis oculata. Bioresour. Technol. 136, 522-528.

Orfield, N.D., Fang, A.J., Valdez, P.J., Nelson, M.C., Savage, P.E., Lin, X.N., Keoleian, G.A., 2014. Life cycle design of an algal biorefinery featuring hydrothermal liquefaction: Effect of reaction conditions and an alternative pathway including microbial regrowth. ACS Sustain. Chem. Eng. 2, 867874 .

Orosz, M.S., Forney, D., 2008. A comparison of algae to biofuel conversion pathways for energy storage off-grid. Report 2.62. Massachusetss Institute of technology. 
Pervez, S., Aman, A., Iqbal, S., Siddiqui, N.N., Qader, S.A.U., 2014. Saccharification and liquefaction of cassava starch: an alternative source for the production of bioethanol using amylolytic enzymes by double fermentation process. BMC Biotechnol. 14, 1-10.

Pham, M., Schideman, L., Scott, J., Rajagopalan, N., Plewa, M.J., 2013. Chemical and biological characterization of wastewater generated from hydrothermal liquefaction of Spirulina. Environ. Sci. Technol. 47, 2131-2138.

Piechocki, J., Zdanis, D., Norris, L., Rakitsky, W., 2011. US20110256282 Lipid-rich microalgal flour food compositions.

Pirwitz, K., Flassig, R.J., Rihko-Struckmann, L.K., Sundmacher, K., 2015a. Energy and operating cost assessment of competing harvesting methods for D. salina in a $\beta$-carotene production process. Algal Res. 12, 161-169.

Pirwitz, K., Rihko-Struckmann, L.K., Sundmacher, K., 2015b. Comparison of flocculation methods for harvesting Dunaliella. Bioresour. Technol. 196, $145-152$.

Richmond, A., 2007. Handbook of microalgal culture biotechnology and applied phycology. Blackwell Science, Oxford.

Srokol, Z., Bouche, A.G., van Estrik, A., Strik, R.C.J., Maschmeyer, T., Peters, J.A., 2004. Hydrothermal upgrading of biomass to biofuel; studies on some monosaccharide model compounds. Carbohydr. Res. 339, 17171726. 
Stanier, R.Y., Kunisawa, R., Mandel, M., Cohen-Bazire, G., 1971. Purification and properties of unicellular blue-green algae (order chroococcales). Bacteriol. Rev. 35, 171-205.

Toor, S.S., Reddy, H., Deng, S., Hoffmann, J., Spangsmark, D., Madsen, L.B., Holm-Nielsen, J.B., Rosendahl, L.A., 2013. Hydrothermal liquefaction of Spirulina and Nannochloropsis salina under subcritical and supercritical water conditions. Bioresour. Technol. 131, 413-419.

Yang, C., Jia, L., Chen, C., Liu, G., Fang, W., 2011. Bio-oil from hydroliquefaction of Dunaliella salina over Ni/REHY catalyst. Bioresour. Technol. 102, 4580-4584.

Yang, W., Li, X., Li, Z., Tong, C., Feng, L., 2015. Understanding lowlipid algae hydrothermal liquefaction characteristics and pathways through hydrothermal liquefaction of algal major components: Crude polysaccharides, crude proteins and their binary mixtures. Bioresour. Technol. 196, 99-108.

Yang, Y.F., Feng, C.P., Inamori, Y., Maekawa, T., 2004. Analysis of energy conversion characteristics in liquefaction of algae. Resour. Conserv. Recy. $43,21-33$.

Yu, G., Zhang, Y., Schideman, L., Funk, T.L., Wang, Z., 2011. Hydrothermal liquefaction of low lipid content microalgae into bio-crude oil. Trans. ASABE 54, 239-246. 


\section{Figure captions}

Figure 1: Influence of reaction time on hydrothermal liquefaction yields and biomass conversion of lipid extracted D. salina biomass. Mild liquefaction was carried out for $0 \mathrm{~min}, 30 \mathrm{~min}$ and $60 \mathrm{~min}$ at 160,180 and $200^{\circ} \mathrm{C}$, respectively.

Figure 2: Influence of reaction temperature on hydrothermal liquefaction yields and biomass conversion of lipid extracted D. salina biomass. Mild liquefaction was carried out for 0 min at 100, 120, 140, 160, 180 and $200^{\circ} \mathrm{C}$, respectively.

Figure 3: Growth and glucose consumption of a) E. coli, b) C. vulgaris and S. cerevisiae on standard culture medium and medium where the glucose concentration was adjusted by addition of the aqueous phase of liquefied D. salina $\left(100^{\circ} \mathrm{C}, 0 \mathrm{~min}\right)$. Cultivation was carried out in duplicates of shaking flasks. Error bars represent the deviation of the measurements from the average value.

Figure 4: Unit scale a) energy demand and b) operating cost calculation per day for industrial $D$. salina based $\beta$-carotene and glucose production. c) Conversion of remnant biomass into glucose after mild hydrothermal liquefaction (HTL). Error bars are based on Monte Carlo simulation to consider the uncertainties of experimental and literature data. 


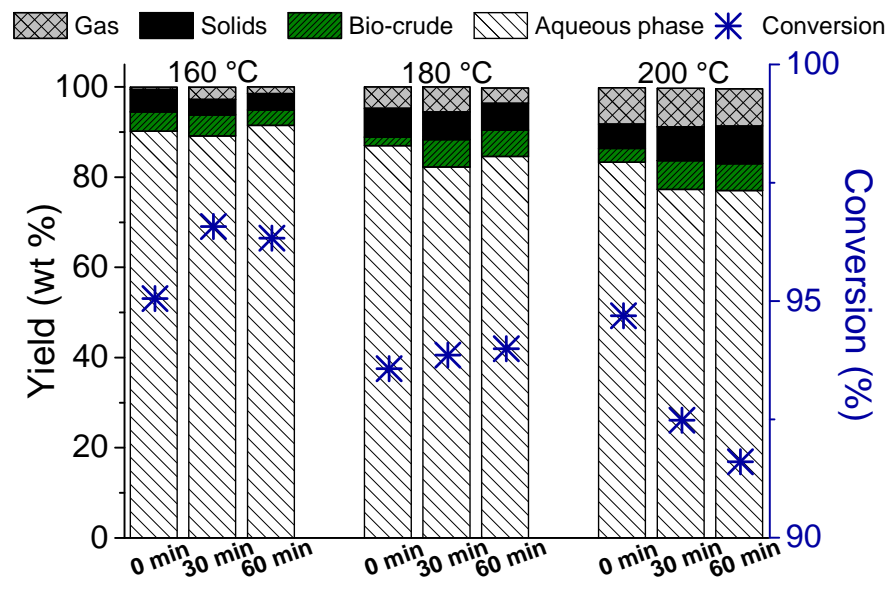

Figure 1 


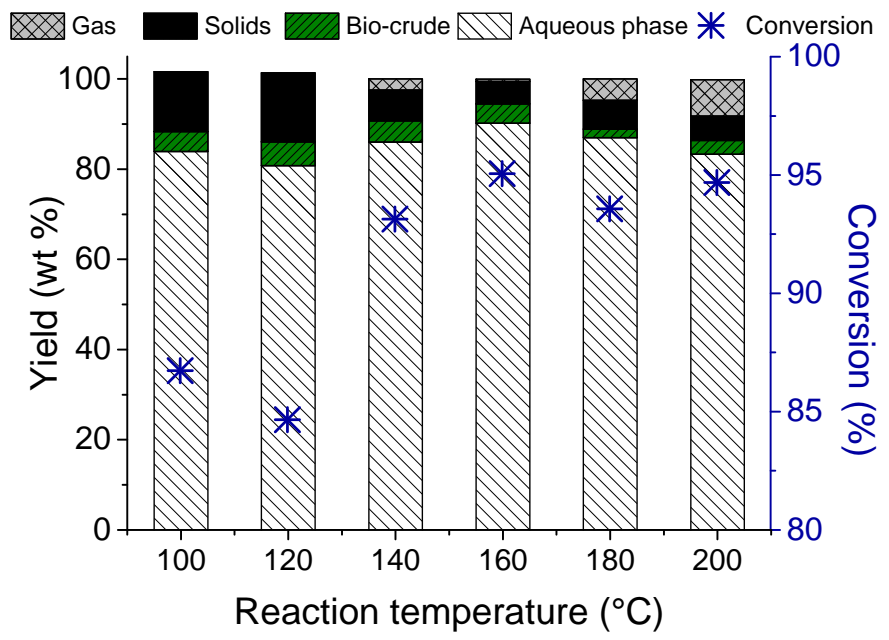

Figure 2 
a)

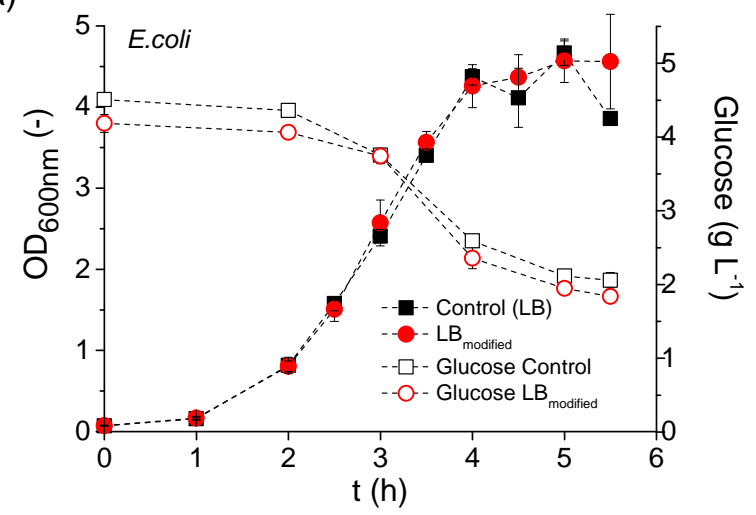

b)

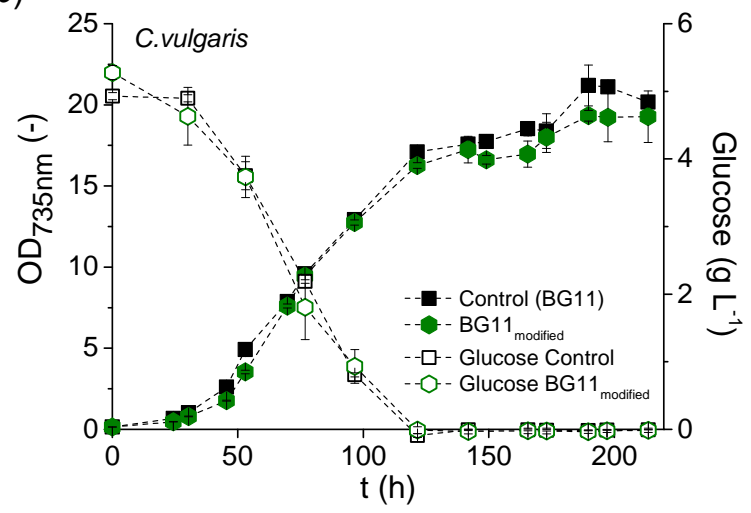

c)

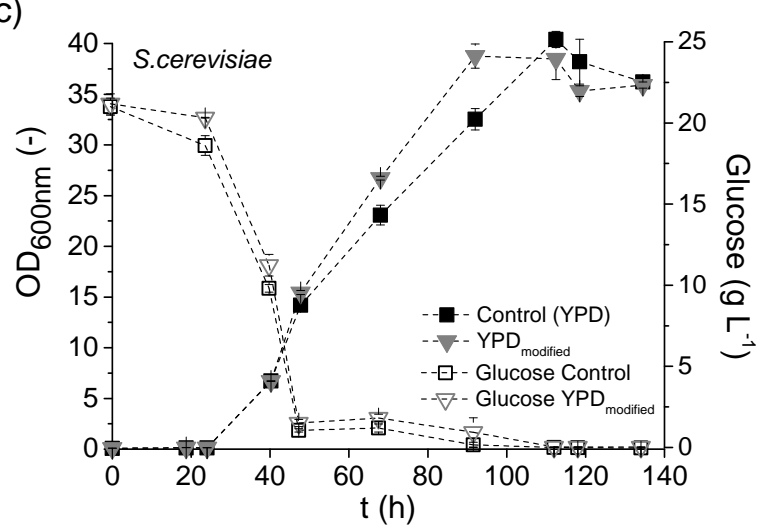

Figure 3 


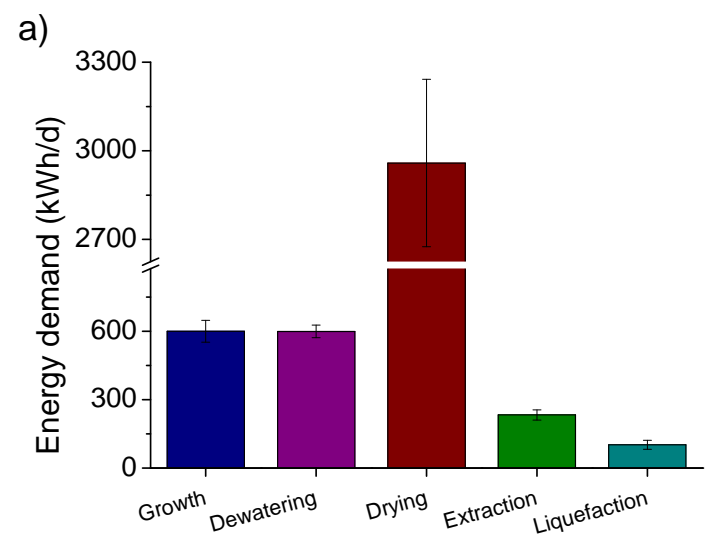

b)

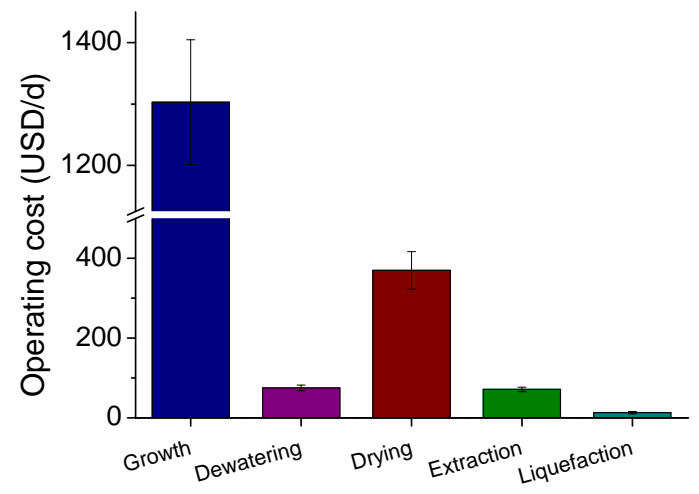

c)

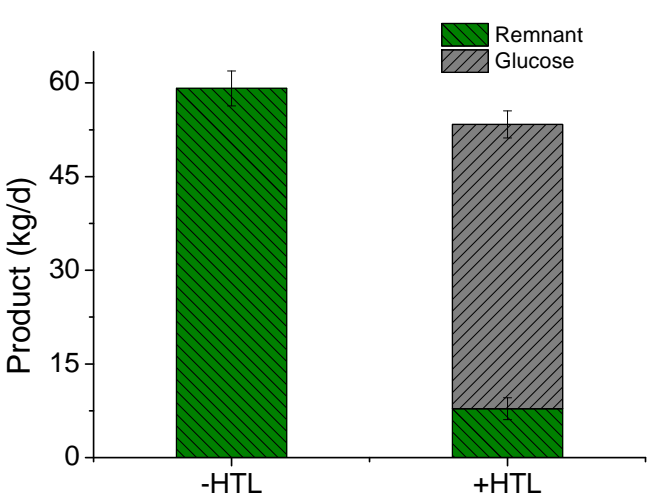

Figure 4 
Table 1: Biomass composition of D. salina powder

\begin{tabular}{lr}
\hline \multicolumn{2}{l}{ Biochemical composition (wt\%) } \\
\hline Carbohydrate & $85.58 \pm 4.98$ \\
(Lipid/Carotinoid $^{a}$ & $11.47 / 4.46 \pm 1.84 / 0.11)$ \\
Protein & $8.46 \pm 0.96$ \\
Moisture & $1.26 \pm 0.01$ \\
Ash & $0.03 \pm 0.002$ \\
\hline Elemental composition (wt\%) & \\
\hline $\mathrm{C}$ & $42.5 \pm 0.15$ \\
$\mathrm{H}$ & $6.85 \pm 0.5$ \\
$\mathrm{~N}$ & $<0.2$ \\
$\mathrm{O}^{b}$ & 50.34 \\
$\mathrm{~S}$ & $<0.01$ \\
\hline
\end{tabular}

a lipid and carotenoid content in the biomass before extraction

${ }^{b}$ calculated by difference 
Table 2: Anion concentrations in the aqueous phase of hydrothermally treated $D$. salina.

\begin{tabular}{ccccccc}
\hline $\mathbf{T}$ & $\mathbf{t}$ & $\mathrm{Na}^{+}$ & $\mathrm{NH}_{4}^{+}$ & $\mathbf{K}^{+}$ & $\mathbf{C a}^{2+}$ & $\mathbf{M g}^{2+}$ \\
${ }^{\circ} \mathrm{C}$ & $\mathrm{min}$ & $\mathrm{mg} \mathrm{g}_{d w}^{-1}$ & $\mathrm{mg} \mathrm{g}_{d w}^{-1}$ & $\mathrm{mg} \mathrm{g}_{d w}^{-1}$ & $\mathrm{mg} \mathrm{g}_{d w}^{-1}$ & $\mathrm{mg} \mathrm{g}_{d w}^{-1}$ \\
\hline 100 & 0 & 0.16 & 0.01 & 0.18 & 0.41 & 0.13 \\
\hline 120 & 0 & 0.14 & 0.01 & 0.15 & 0.35 & 0.11 \\
\hline 140 & 0 & 0.15 & 0.01 & 0.14 & 0.32 & 0.15 \\
\hline 160 & 0 & 0.39 & 0.01 & 0.20 & 0.61 & 0.18 \\
& 30 & 0.32 & 0.01 & 0.30 & 1.00 & 0.22 \\
& 60 & 0.15 & 0.02 & 0.22 & 1.08 & 0.21 \\
\hline 180 & 0 & 0.15 & 0.01 & 0.25 & 0.86 & 0.21 \\
& 30 & 0.13 & 0.01 & 0.21 & 0.73 & 0.18 \\
& 60 & 0.24 & 0.02 & 0.16 & 0.62 & 0.15 \\
\hline 200 & 0 & 0.17 & 0.01 & 0.22 & 0.84 & 0.22 \\
& 30 & 0.28 & 0.03 & 0.29 & 1.06 & 0.26 \\
& 60 & 0.31 & 0.02 & 0.33 & 1.04 & 0.24 \\
\hline
\end{tabular}


Table 3: Cation concentrations in the aqueous phase of hydrothermally treated D. salina.

\begin{tabular}{ccccccccc}
\hline $\mathbf{T}$ & $\mathbf{t}$ & Acetate & Formate & $\mathbf{C l}^{-}$ & $\mathrm{NO}_{2}^{-}$ & $\mathrm{NO}_{3}^{-}$ & $\mathbf{P O}_{4}^{3-}$ & $\mathbf{S O}_{4}^{2-}$ \\
${ }^{\circ} \mathrm{C}$ & $\mathrm{min}$ & $\mathrm{mg} \mathrm{g}_{d w}^{-1}$ & $\mathrm{mg} \mathrm{g}_{d w}^{-1}$ & $\mathrm{mg} \mathrm{g}_{d w}^{-1}$ & $\mathrm{mg} \mathrm{g}_{d w}^{-1}$ & $\mathrm{mg} \mathrm{g}_{d w}^{-1}$ & $\mathrm{mg} \mathrm{g}_{d w}^{-1}$ & $\mathrm{mg} \mathrm{g}_{d w}^{-1}$ \\
\hline 100 & 0 & 0.70 & 2.24 & 0.42 & 0.04 & 0.45 & 0.52 & 0.52 \\
\hline 120 & 0 & 0.60 & 1.92 & 0.36 & 0.03 & 0.39 & 0.45 & 0.45 \\
\hline 140 & 0 & 0.86 & 3.23 & 0.35 & 0.03 & 0.36 & 0.57 & 0.47 \\
\hline 160 & 0 & 1.12 & 1.96 & 1.04 & 0.05 & 0.68 & 0.83 & 0.74 \\
& 30 & 2.95 & 4.86 & 0.80 & 0.05 & 0.67 & 0.75 & 0.77 \\
& 60 & 2.79 & 4.50 & 0.56 & 0.06 & 0.67 & 0.76 & 0.73 \\
\hline 180 & 0 & 5.30 & 15.97 & 0.63 & 0.06 & 0.74 & 0.88 & 0.82 \\
& 30 & 4.48 & 13.50 & 0.53 & 0.05 & 0.63 & 0.74 & 0.69 \\
& 60 & 4.01 & 10.42 & 0.54 & 0.05 & 0.57 & 0.67 & 0.86 \\
\hline 200 & 0 & 1.49 & 2.30 & 0.41 & 0.04 & 0.51 & 0.59 & 0.54 \\
& 30 & 3.67 & 9.95 & 0.73 & 0.06 & 0.74 & 0.88 & 0.92 \\
& 60 & 3.82 & 12.13 & 0.71 & 0.06 & 0.79 & 0.96 & 0.90 \\
\hline
\end{tabular}


Table 4: Glucose yields in the aqueous phase of hydrothermally treated D. salina.

\begin{tabular}{ccc}
\hline $\begin{array}{c}\mathbf{T} \\
{ }^{\circ} \mathrm{C}\end{array}$ & $\begin{array}{c}\text { Glucose } \\
\text { min }\end{array}$ & wt\% \\
\hline 100 & 0 & $77.01 \pm 0.39$ \\
\hline 120 & 0 & $66.47 \pm 5.82$ \\
\hline 140 & 0 & $79.54 \pm 1.18$ \\
\hline 160 & 0 & $68.40 \pm 4.43$ \\
& 30 & $62.49 \pm 0.74$ \\
& 60 & $68.73 \pm 1.12$ \\
\hline 180 & 0 & $59.44 \pm 1.35$ \\
& 30 & $65.68 \pm 3.14$ \\
& 60 & $52.67 \pm 0.37$ \\
\hline 200 & 0 & $69.63 \pm 1.39$ \\
& 30 & $69.08 \pm 0.60$ \\
& 60 & $66.54 \pm 0.50$ \\
\hline
\end{tabular}




\section{Supplementary materials}

Valorization of the aqueous phase obtained from hydrothermally treated Dunaliella salina remnant biomass

Kristin Pirwitz, Liisa Rihko-Struckmann, Kai Sundmacher

Table S1: Carbohydrate yields in the aqueous phase of hydrothermally treated $D$. salina.

\begin{tabular}{ccccc}
\hline $\mathbf{T}$ & $\mathbf{t}$ & Galactose & Fructose & Sucrose \\
${ }^{\circ} \mathrm{C}$ & min & wt\% & wt\% & wt $\%$ \\
\hline 100 & 0 & n.d. & n.d. & n.d. \\
\hline 120 & 0 & n.d. & n.d. & n.d. \\
\hline 140 & 0 & n.d. & n.d. & n.d. \\
\hline 160 & 0 & n.d. & n.d. & $2.93 \pm 0.01$ \\
& 30 & $1.32 \pm 0.00$ & n.d. & $1.02 \pm 0.17$ \\
& 60 & $1.60 \pm 0.66$ & n.d. & n.d. \\
\hline 180 & 0 & $1.60 \pm 0.18$ & $0.37 \pm 0.12$ & n.d. \\
& 30 & $2.41 \pm 0.04$ & n.d. & n.d. \\
& 60 & $0.38 \pm 0.03$ & $0.47 \pm 0.24$ & n.d. \\
\hline 200 & 0 & $2.70 \pm 0.05$ & n.d. & $1.00 \pm 0.46$ \\
& 30 & $5.50 \pm 0.37$ & n.d. & $5.30 \pm 2.09$ \\
& 60 & $3.66 \pm 0.25$ & n.d. & $3.73 \pm 1.21$ \\
\hline
\end{tabular}

